\title{
Derivation of Hydrodynamics from the Hamiltonian Description of Particle Systems
}

\section{$\operatorname{AUTHOR}(\mathrm{S}):$}

Sasa, Shin-ichi

\section{CITATION:}

Sasa, Shin-ichi. Derivation of Hydrodynamics from the Hamiltonian Description of Particle Systems. Physical Review Letters 2014, 112(10): 100602.

\section{ISSUE DATE:}

2014-03

URL:

http://hdl.handle.net/2433/187039

\section{RIGHT:}

(C) 2014 American Physical Society 


\title{
Derivation of Hydrodynamics from the Hamiltonian Description of Particle Systems
}

\author{
Shin-ichi Sasa \\ Department of Physics, Kyoto University, Kyoto 606-8502, Japan \\ (Received 23 June 2013; revised manuscript received 4 February 2014; published 14 March 2014)

\begin{abstract}
Hamiltonian particle systems may exhibit nonlinear hydrodynamic phenomena as the time evolution of the density fields of energy, momentum, and mass. In this Letter, an exact equation describing the time evolution is derived assuming the local Gibbs distribution at initial time. The key concept in the derivation is an identity similar to the fluctuation theorems. The Navier-Stokes equation is obtained as a result of simple perturbation expansions in a small parameter that represents the scale separation.
\end{abstract}

DOI: 10.1103/PhysRevLett.112.100602

PACS numbers: 05.20.Jj, 05.70.Ln, 47.10.-g

Introduction.-Let us consider a large number of interacting particles that obey a classical Hamiltonian equation as an isolated system. The initial condition is assumed to provide spatial variation in the macroscopic density fields. Examples include turbulent configurations of the momentum density field. Through time evolution of the particles, the density fields exhibit interesting space-time structures, and the system eventually relaxes to an equilibrium state. This process is believed to be described universally by hydrodynamic equations of the density fields for liquids and gases [1]. As a stimulating numerical experiment of a Hamiltonian particle system, quite recently, the Kolmogorov spectrum has been observed in a transient state [2]. However, it remains unclear whether the hydrodynamic description of such violent time evolution can be understood on the basis of Hamiltonian particle systems.

The problem of deriving the hydrodynamic equations has been studied over the last century by various approaches such as the analysis of the Boltzmann equation [3], the calculation on the basis of local equilibrium distributions [4,5], and the derivation from hypothetical nonequilibrium ensembles [6-9]. However, nonlinear hydrodynamic phenomena of liquids are out of the scope of these classical works, because the Boltzmann equation applies to only dilute gases, the local equilibrium distributions cannot describe dissipation effects, and the hypothetical ensembles are not justified beyond the linear response regime from a global equilibrium state [10].

Nevertheless, a physical intuition of the hydrodynamic description in particle systems is simple, and it has been known as follows. When the length scale of the spatial variation of the density fields is much larger than the particle scales, the particle distribution may be characterized by a local equilibrium distribution with local thermodynamic variables. If such local equilibrium propagates in time, the solution of the Liouville equation may be close to the local equilibrium distribution [11]. For example, turbulence is a strongly nonlinear phenomenon described by a deterministic equation for the density fields, whereas the particle distribution in the turbulent state is still near local equilibrium. Thus, the nonlinear hydrodynamic equations may be derived by developing a perturbation theory with a small parameter representing the scale separation.

As one attempt that follows the physical intuition, a perturbation theory leading to the incompressible NavierStokes equation was presented with the assumption of the propagation of local equilibrium [12]. Since this study attempts to construct solutions of the Liouville equation without specifying the initial condition, it seems difficult to investigate the validity of the assumption. Rather, in order to obtain a definite result, it may be necessary that a special class of initial conditions is focused on.

Such a situation can be seen in the statistical mechanical derivation of the second law of thermodynamics. If an initial distribution satisfies some conditions, the second law of thermodynamics can be derived [13,14]. In particular, if the canonical distribution is assumed initially, the proof of the second law becomes quite simple by utilizing the Jarzynski equality [15]. These results obtained for special but familiar initial distributions provide key insights into the second law of thermodynamics.

In this Letter, as an extension of these studies, the nonlinear hydrodynamic equations are derived in a quite compact manner for an isolated Hamiltonian system (i.e., a purely mechanical system) with an initial condition given by the local Gibbs distribution. The crucial step in the derivation is to find a universal relation similar to the fluctuation theorems [16-23], by which an exact expression for the time evolution of the density fields is obtained. Furthermore, a straightforward perturbation leads to the Navier-Stokes equation after introducing a scale separation parameter that is assumed to be small.

Framework.-Let $\boldsymbol{r}_{i}$ and $\boldsymbol{p}_{i}(1 \leq i \leq N)$ be the position and the momentum of $i$ th particle with the mass $m$. The short-range interaction potential between $i$ th and $j$ th particles is expressed as $V\left(\left|\boldsymbol{r}_{i j}\right|\right)$, where $\boldsymbol{r}_{i j}=\boldsymbol{r}_{i}-\boldsymbol{r}_{j}$. The phase space coordinate of the system is a collection of $\left(\boldsymbol{r}_{i}, \boldsymbol{p}_{i}\right)$, which is denoted by $\Gamma$. The particles are confined in a cube $\Omega$ with length $L$. For simplicity, periodic boundary conditions are assumed. The time evolution of 
the system is described by the Hamiltonian equation. The solution of the equation for an initial state $\Gamma$ is simply denoted as $\Gamma_{t}$ for any real number $t$. That is, $\Gamma_{t+s}=\left(\Gamma_{t}\right)_{s}$ for any $t$ and $s$.

There are five conserved quantities: the total mass, the total momentum, and the total energy. It is assumed that there are no other independent conserved quantities and that there are no soft modes arising from symmetry breaking. Corresponding to the conserved quantities, the following microscopic density fields are defined: $\hat{\rho}(\boldsymbol{r} ; \Gamma) \equiv \sum_{i} m \delta\left(\boldsymbol{r}-\boldsymbol{r}_{i}\right), \hat{\boldsymbol{\pi}}(\boldsymbol{r} ; \Gamma) \equiv \sum_{i} \boldsymbol{p}_{i} \delta\left(\boldsymbol{r}-\boldsymbol{r}_{i}\right)$, and

$$
\hat{h}(\boldsymbol{r} ; \Gamma) \equiv \sum_{i}\left[\frac{p_{i}^{2}}{2 m}+\frac{1}{2} \sum_{j \neq i} V\left(\left|\boldsymbol{r}_{i j}\right|\right)\right] \delta\left(\boldsymbol{r}-\boldsymbol{r}_{i}\right) .
$$

A collection of the five density fields is denoted by $\hat{C}=(\hat{h}, \hat{\boldsymbol{\pi}}, \hat{\rho})$. A component of $\hat{C}$ is expressed as $\hat{C}^{\alpha}$, where $\alpha \in\{0,1,2,3,4\}$. The density fields $\hat{C}^{\alpha}(\boldsymbol{r} ; \Gamma)$ satisfy the continuity equation

$$
\partial_{t} \hat{C}^{\alpha}\left(\boldsymbol{r} ; \Gamma_{t}\right)+\partial^{a} \hat{J}^{\alpha a}\left(\boldsymbol{r} ; \Gamma_{t}\right)=0
$$

where the microscopic currents $\hat{J}^{\alpha a}(\boldsymbol{r} ; \Gamma)$ can be written explicitly in terms of $\Gamma$ [24]. The roman alphabet $a, b$, and $c$ take values 1,2 , or 3 , which represents the index of the Euclidian coordinates. Here and hereafter, the same index appearing in one term indicates that the summation with respect to this index is taken. For the sake of notation simplicity, $f \cdot g \equiv \int_{\Omega} d^{3} \boldsymbol{r} f(\boldsymbol{r}) g(\boldsymbol{r})$.

The main assumption in this Letter is that initial states $\Gamma$ at $t=0$ are chosen according to the local Gibbs (LG) distribution

$$
P_{\mathrm{LG}}(\Gamma ; \lambda)=e^{-\lambda^{\alpha} \cdot \hat{C}^{\alpha}(\Gamma)-\Psi(\lambda)},
$$

where the position dependent parameter $\lambda(\boldsymbol{r})$ characterizes the inhomogeneous distribution of the density fields. The normalization condition of the probability determines the functional $\Psi(\lambda)$ for the Hamiltonian. Let us call $\lambda$ and $\Psi(\lambda)$ the conjugate field and the Mathieu functional, respectively, although these names may not be accurate except for the limited case where $\lambda$ is spatially homogeneous. $\Psi(\lambda)$ is assumed to be a convex functional of $\lambda$ as a natural extension from the spatially homogeneous case. One example of initial states may be a snapshot of the molecular configuration in a turbulent state.

The local Gibbs distribution describes an inhomogeneous equilibrium state in the moving frame with the local velocity $\boldsymbol{u} \equiv-\lambda / \lambda^{0}$. Indeed, let $\Gamma^{\prime}$ be the phase space coordinate obtained from $\Gamma$ by transforming $\boldsymbol{p}_{i}$ in $\Gamma$ to $\boldsymbol{p}_{i}-\boldsymbol{m} \boldsymbol{u}\left(\boldsymbol{r}_{i}\right)$. In general, a dynamical variable in the moving frame is defined as $\hat{A}^{\prime}(\Gamma) \equiv \hat{A}\left(\Gamma^{\prime}\right)$ for any dynamical variable $\hat{A}$. Then, $\lambda^{\alpha} \cdot \hat{C}^{\alpha}$ is written as

$$
\lambda^{\alpha} \cdot \hat{C}^{\alpha}=\lambda^{0} \cdot \hat{h}^{\prime}+\left(\lambda^{4}-\frac{1}{2} \frac{|\lambda|^{2}}{\lambda^{0}}\right) \cdot \hat{\rho}
$$

which implies that $\lambda^{0}(\boldsymbol{r})$ and $\lambda^{4}(\boldsymbol{r})$ are related to inhomogeneous inverse temperature and a one-body potential function. It should be noted that $\hat{h}^{\prime}$ is interpreted as the internal energy density field.

The expectation value of the density field $\hat{C}\left(r ; \Gamma_{t}\right)$ at time $t$ with respect to the initial distribution is given by $\int d \Gamma P_{\mathrm{LG}}(\Gamma ; \lambda) \hat{C}\left(\boldsymbol{r} ; \Gamma_{t}\right)$. It is further written as $\int d \Gamma P_{t}(\Gamma) \hat{C}(\boldsymbol{r} ; \Gamma)$, where

$$
P_{t}(\Gamma)=e^{-\lambda^{\alpha} \cdot \hat{C}^{\alpha}\left(\Gamma_{-t}\right)-\Psi(\lambda)}
$$

using the Liouville theorem $\left|d \Gamma_{t} / d \Gamma\right|=1$. The expectation values with respect to $P_{\mathrm{LG}}(\Gamma ; \lambda)$ and $P_{t}(\Gamma)$ are denoted by \langle\rangle$_{\lambda}^{\mathrm{LG}}$ and \langle\rangle$_{t}$, respectively. Then, the time evolution of $C_{t}^{\alpha}(\boldsymbol{r})$, which represents $\left\langle\hat{C}^{\alpha}(\boldsymbol{r})\right\rangle_{t}$, is derived as

$$
\partial_{t} C_{t}^{\alpha}(\boldsymbol{r})+\partial^{a} \boldsymbol{J}_{t}^{\alpha a}(\boldsymbol{r})=0,
$$

where $J_{t}^{\alpha a}(\boldsymbol{r})$ is found to be equal to $\left\langle\hat{\boldsymbol{J}}^{\alpha a}(\boldsymbol{r})\right\rangle_{t}$. Throughout this Letter, the dynamical variable with the hat symbol, e.g., $\hat{A}$, indicates that this variable depends on the phase space coordinate $\Gamma$, whereas the quantity without the hat symbol indicates an expectation value. When $J_{t}^{\alpha a}$ is expressed in terms of $C_{t}$, (6) becomes a hydrodynamic equation.

Main result.-I present three identities in the framework. The first identity is

$$
\partial^{a} \lambda^{\alpha} \cdot\left\langle\hat{J}^{\alpha a}\right\rangle_{\lambda}^{\mathrm{LG}}=0
$$

for any $\lambda$, which can be derived from the trivial relation $\left.\partial_{t}\left[\int d \Gamma P_{t}(\Gamma)\right]\right|_{t=0}=0$.

The second identity originates from statistical mechanical formulas of the local Gibbs distribution. In order to utilize the formulas, the time evolution of the density fields is represented by using the time dependent conjugate fields $\lambda_{t}^{\alpha}$, which is defined by $C_{t}^{\alpha}(\boldsymbol{r})=\left\langle\hat{C}^{\alpha}(\boldsymbol{r})\right\rangle_{\lambda_{t}}^{\mathrm{LG}}$. This definition is then written as

$$
C_{t}^{\alpha}(\boldsymbol{r})=-\left.\frac{\delta \Psi(\lambda)}{\delta \lambda^{\alpha}(\boldsymbol{r})}\right|_{\lambda=\lambda_{t}} .
$$

Owing to the convexity of the Mathieu functional $\Psi(\lambda)$, (8) is further expressed as a variational equation:

$$
\lambda_{t}=\operatorname{Arginf}_{\lambda}\left[\lambda^{\alpha} \cdot C_{t}^{\alpha}+\Psi(\lambda)\right] .
$$

This naturally leads to the definition of the functional

$$
\mathcal{S}(C) \equiv \inf _{\lambda}\left[\lambda^{\alpha} \cdot C^{\alpha}+\Psi(\lambda)\right] .
$$

When the density field $C$ is spatially homogeneous, $\mathcal{S}(C)$ is identical to the thermodynamic entropy. In this sense, $\mathcal{S}$ 
may be an extension of the entropy to that for nonequilibrium states characterized by the density field, but no thermodynamic interpretation is given yet. The important thing here is that the quantity $\mathcal{S}(C)$ is determined from the Hamiltonian under consideration. Then, for a given density field $C_{t}(\boldsymbol{r})$, the conjugate field $\lambda_{t}(\boldsymbol{r})$ is calculated by

$$
\lambda_{t}(\boldsymbol{r})=\left.\frac{\delta \mathcal{S}(C)}{\delta C(\boldsymbol{r})}\right|_{C=C_{t}},
$$

which is the second identity. At this stage, let us remember that $C_{t}$ and $\lambda_{t}$ are determined uniquely from the time evolution and the initial distribution.

The third identity is a variant of the so-called fluctuation theorems. In order to obtain it, (5) is formally rewritten as

$$
P_{t}(\Gamma)=e^{-\lambda_{t}^{\alpha} \cdot \hat{C}^{\alpha}(\Gamma)-\Psi\left(\lambda_{t}\right)+\hat{\Sigma}_{t}(\Gamma)},
$$

where

$$
\hat{\Sigma}_{t}(\Gamma)=\int_{0}^{t} d s \partial_{s}\left[\lambda_{s}^{\alpha} \cdot \hat{C}^{\alpha}\left(\Gamma_{s-t}\right)+\Psi\left(\lambda_{s}\right)\right] .
$$

By comparing (12) with (5), it is found for any dynamical variable $\hat{A}(\Gamma)$ that

$$
\langle\hat{A}\rangle_{t}=\left\langle\hat{A} e^{\hat{\Sigma}_{t}}\right\rangle_{\lambda_{t}}^{\mathrm{LG}},
$$

which is the third identity. Various nontrivial relations of statistical quantities can be derived from this identity. As one example, (14) with $\hat{A}=e^{-\hat{\Sigma}_{t}}$ becomes $\left\langle e^{-\hat{\Sigma}_{t}}\right\rangle_{t}=1$, which corresponds to an integral fluctuation theorem. With a trivial inequality $e^{-x} \geq 1-x$, the integral fluctuation theorem leads to the inequality $\left\langle\hat{\Sigma}_{t}\right\rangle_{t} \geq 0$. Since (13) gives $\left\langle\Sigma_{t}\right\rangle_{t}=\mathcal{S}\left(C_{t}\right)-\mathcal{S}\left(C_{0}\right),\left\langle\hat{\Sigma}_{t}\right\rangle_{t} \geq 0$ implies $\mathcal{S}\left(C_{t}\right) \geq \mathcal{S}\left(C_{0}\right)$ for any $t$. This can be related to the second law of thermodynamics for some special cases.

These three identities provide a useful representation of the time evolution. Given $C_{s}$ for $s \in[0, t]$, one determines $\lambda_{s}$ by the second identity (11). Then, the third identity (14) leads to

$$
\boldsymbol{J}_{t}^{\alpha a}(\boldsymbol{r})=\left\langle\hat{\boldsymbol{J}}^{\alpha a}(\boldsymbol{r}) e^{\hat{\Sigma}_{t}}\right\rangle_{\lambda_{t}}^{\mathrm{LG}}
$$

with

$$
\hat{\Sigma}_{t}(\Gamma)=\int_{0}^{t} d s\left[\left(\partial^{a} \lambda_{s}^{\alpha}\right) \cdot \delta \hat{J}_{s}^{\alpha a}+\left(\partial_{s} \lambda_{s}^{\alpha}\right) \cdot \delta \hat{C}_{s}^{\alpha}\right],
$$

where $\delta \hat{A}_{s} \equiv \hat{A}\left(\Gamma_{s-t}\right)-\langle\hat{A}\rangle_{\lambda_{s}}^{\mathrm{LG}}$ for any $\hat{A}$, and the first identity (7) and the formula (8) have been used in the derivation of (16). The continuity equation (6) leads to $\partial_{t} C_{t}^{\alpha}(\boldsymbol{r})$ from $J_{t}^{\alpha a}(\boldsymbol{r})$. Expressions similar to the nonequilibrium ensemble (12) with (16) have been proposed in many studies such as Refs. [7-9]. (See Ref. [10] for the rigorous characterization on the so-called Mclennan ensemble.) However, in this Letter, purely Hamiltonian systems are considered without interaction with external reservoirs, in contrast to previous studies.

It should be stressed that the general result consisting of (6), (11), (15), and (16) can be obtained without any complicated calculation. This exact evolution equation of $C_{t}$ will be a starting point for the analysis of all hydrodynamic phenomena which may include nonstandard cases that are not described by the Navier-Stokes equation. Moreover, this expression immediately leads to the wellknown hydrodynamic equations when a small parameter representing the scale separation is introduced, which is explained below.

Perturbation theory.-Let $\xi_{\text {micro }}$ be the maximum length scale appearing in the molecular description such as the molecule size, the interaction length, or the mean free path, and $\xi_{\text {macro }}$ be the minimum length characterizing macroscopic behaviors. A scale separation parameter is then introduced as $\epsilon \equiv \xi_{\text {micro }} / \xi_{\text {macro }}$, which is assumed to be small. Hereafter, all of the quantities are assumed to be dimensionless by setting $\xi_{\text {micro }}=1, m=1$, and $\lambda^{0}\left(\boldsymbol{r}_{0}\right)=1$ for some $\boldsymbol{r}_{0} \in \Omega$.

Macroscopic density fields are initially generated by choosing $\Gamma$ according to the local Gibbs distribution with the choice $\lambda^{\alpha}(\boldsymbol{r})=\bar{\lambda}^{\alpha}(\epsilon \boldsymbol{r})$, where the functional form of $\bar{\lambda}^{\alpha}$ is independent of $\epsilon$. All the small quantities originate from this $\epsilon$. For example, $\partial^{a} C_{s}^{\alpha} \simeq O(\epsilon)$ and $\partial^{a} \lambda_{s}^{\alpha} \simeq O(\epsilon)$ are reasonably conjectured for any $s$. Based on these estimations, any quantity $A$ can be expanded as $A=A^{(0)}+A^{(1)}+A^{(2)}+O\left(\epsilon^{3}\right)$, where $A^{(k)} / \epsilon^{k}$ is finite for $\epsilon \rightarrow 0$.

First, the functional $\mathcal{S}$ is expanded. Since $\mathcal{S}$ may be expressed as a space integration of some functions of the density fields and their spatial derivatives, $\mathcal{S}^{(1)}=0$ from the reflection symmetry, $\mathcal{S}^{(2)}$ contains terms such as $\left(\partial^{a} C^{\alpha}\right)^{2}$, and $\mathcal{S}^{(0)}$ consists of terms without spatial derivatives. That is, $\mathcal{S}^{(0)}$ is equal to the space integration of the local entropy density, where (4) and (10) should be noticed. Explicitly, by using the thermodynamic entropy density $s_{\text {th }}$ as a function of $h^{\prime}$ and $\rho$ for the system, $\mathcal{S}^{(0)}$ is expressed as

$$
\mathcal{S}^{(0)}(C)=\int_{\Omega} d^{3} \boldsymbol{r} s_{\mathrm{th}}\left(h^{\prime}(\boldsymbol{r}), \rho(\boldsymbol{r})\right) .
$$

According to thermodynamics, the inverse temperature and the chemical potential are defined by $\beta \equiv \partial s_{\mathrm{th}}\left(h^{\prime}, \rho\right) / \partial h^{\prime}$ and $\mu \equiv-\beta^{-1} \partial s_{\text {th }}\left(h^{\prime}, \rho\right) / \partial \rho$, respectively. Through this relation, $\beta_{s}(\boldsymbol{r})$ and $\mu_{s}(\boldsymbol{r})$ are determined from $C_{s}^{\alpha}(\boldsymbol{r})$. Then, from (11) and (17), $\beta_{s}$ and $\mu_{s}$ are related to the conjugate fields as $\beta_{s}=\lambda_{s}^{0}+O\left(\epsilon^{2}\right)$ and $\beta_{s} \mu_{s}=$ $-\lambda_{s}^{4}+\beta_{s}\left|\boldsymbol{u}_{s}\right|^{2} / 2+O\left(\epsilon^{2}\right)$, and the straightforward calculation [24] using a thermodynamic relation yields 


$$
\begin{aligned}
\left(\partial^{a} \lambda_{s}^{\alpha}\right) \cdot J_{s}^{\alpha a}= & \left(\partial^{a} \beta_{s}\right) \cdot J_{s}^{0 a \prime}-\left(\beta_{s} \partial^{b} u_{s}^{a}\right) \cdot\left(J_{s}^{a b \prime}-p_{s} \delta^{a b}\right) \\
& +O\left(\epsilon^{3}\right),
\end{aligned}
$$

where $p(\boldsymbol{r})$ is the thermodynamic pressure determined from $s_{\mathrm{th}}\left(h^{\prime}(\boldsymbol{r}), \rho(\boldsymbol{r})\right)$.

Next, since $\hat{\Sigma}_{t}$ in (16) is estimated as $O(\epsilon)$, it is found from (15) that $J_{t}^{\alpha a(0)}=\left\langle\hat{J}^{\alpha a}\right\rangle_{\lambda_{t} \mathrm{LG}}^{\mathrm{L}}$. From the isotropic property, $\left\langle\hat{J}^{0 a^{\prime}}\right\rangle_{\lambda}^{\mathrm{LG}}=0$ and $\left\langle\hat{J}^{a b \prime}\right\rangle_{\lambda}^{\mathrm{L}^{t} \mathrm{G}}=\phi \delta^{a b}$, where $\phi(\boldsymbol{r})$ is a scalar field. Then, the combination of (7) with (18) leads to $\beta \partial^{a} u^{a} \cdot(\phi-p)=0$. Since $\phi-p$ is independent of $\boldsymbol{u}, \phi=$ $p$ is obtained. The result was in fact derived by the direct calculation [4]. It is thus concluded that $J^{0 a(0)}=$ $(h+p) u^{a}, J^{a b(0)}=p \delta^{a b}+\rho u^{a} u^{b}$, and $J^{4 a}=J^{4 a(0)}=\rho u^{a}$, where the relation $\hat{J}^{\alpha a \prime}$ and $\hat{J}^{\alpha a}$ has been used [24]. The hydrodynamic equation $\partial_{t} C_{t}^{\alpha}+\partial^{a} J_{t}^{\alpha a(0)}=0$ is the Euler equation. (See Ref. [25] for the mathematical derivation of the Euler equation.)

Furthermore, from (15), it is found that $J_{t}^{\alpha a(1)}=$ $\left\langle\hat{J}^{\alpha a} \hat{\Sigma}_{t}^{(1)}\right\rangle_{\lambda_{t}}^{\mathrm{LG}}$, where $\hat{\Sigma}_{t}^{(1)}$ represents the estimation that $\partial_{s} \lambda_{s}$ in $\hat{\Sigma}_{t}$ is given by the Euler equation. Then, $\hat{\Sigma}_{t}^{(1)}$ is calculated as [24]

$$
\hat{\Sigma}_{t}^{(1)}(\Gamma)=\int_{0}^{t} d s\left[\left(\partial^{a} \beta_{s}\right) \cdot \hat{q}^{a}\left(\Gamma_{s-t}\right)-\left(\beta_{s} \partial^{a} u_{s}^{b}\right) \cdot \hat{\tau}^{a b}\left(\Gamma_{s-t}\right)\right],
$$

where

$$
\begin{gathered}
\hat{q}^{a} \equiv \hat{J}^{0 a \prime}-\frac{h^{\prime}+p}{\rho} \hat{\pi}^{a \prime} \\
\hat{\tau}^{a b} \equiv \delta \hat{J}^{a b \prime}-\left[\left(\frac{\partial p}{\partial h^{\prime}}\right)_{\rho} \delta \hat{h}^{\prime}+\left(\frac{\partial p}{\partial \rho}\right)_{h^{\prime}} \delta \hat{\rho}\right] \delta^{a b} .
\end{gathered}
$$

$\hat{q}^{a}$ and $\hat{\tau}^{a b}$ are interpreted as the irreducible part of the energy and momentum fluxes in the moving frame with the local velocity, in which the contribution from fluctuations of the density fields are subtracted from the energy and momentum fluxes. By combining (20) and (21) with the relation between $\hat{J}^{\alpha a \prime}$ and $\hat{J}^{\alpha a}$, it is obtained that $J_{t}^{0 a(1)}=$ $\tau_{t}^{a b(1)} u_{t}^{b}+q_{t}^{a(1)}$ and $J_{t}^{a b(1)}=\tau_{t}^{a b(1)}$. Here, it is assumed that the characteristic length and time scales of $C^{\alpha}$ are much larger than the correlation length and time of $\hat{\tau}^{a b(1)}$ and $\hat{q}^{a(1)}$. This enables us to replace $C_{s}^{\alpha}\left(\boldsymbol{r}^{\prime}\right)$ in the space-time integration of $\left\langle\hat{q}^{a(1)} \hat{\Sigma}_{t}^{(1)}\right\rangle_{\lambda_{t}}^{\mathrm{LG}}$ and $\left\langle\hat{\tau}^{a b(1)} \hat{\Sigma}_{t}^{(1)}\right\rangle_{\lambda_{t}}^{\mathrm{LG}}$ by $C_{t}^{\alpha}(\boldsymbol{r})$. By noting the isotropic property of the system, it is finally derived that $q_{t}^{a(1)}=\kappa\left(\partial^{a} \beta_{t}\right)$ and

$$
\tau_{t}^{a b(1)}=-\eta\left(\partial^{a} u_{t}^{b}+\partial^{b} u_{t}^{a}\right)-\left(\zeta-\frac{2}{3} \eta\right) \partial^{c} u_{t}^{c} \delta^{a b},
$$

with the Green-Kubo formula [26]

$$
\begin{gathered}
\kappa=\int_{0}^{t} d s \int d^{3} \boldsymbol{r}^{\prime}\left\langle\hat{q}^{1}\left(\boldsymbol{r}^{\prime} ; \Gamma_{s-t}\right) \hat{q}^{1}(\boldsymbol{r} ; \Gamma)\right\rangle_{\lambda_{t}}^{\mathrm{LG}}, \\
\eta=\beta_{t} \int_{0}^{t} d s \int d^{3} \boldsymbol{r}^{\prime}\left\langle\hat{\tau}^{12}\left(\boldsymbol{r}^{\prime} ; \Gamma_{s-t}\right) \hat{\tau}^{12}(\boldsymbol{r} ; \Gamma)\right\rangle_{\lambda_{t}}^{\mathrm{LG}}, \\
\zeta=\beta_{t} \int_{0}^{t} d s \int d^{3} \boldsymbol{r}^{\prime}\left\langle\delta \hat{p}\left(\boldsymbol{r}^{\prime} ; \Gamma_{s-t}\right) \delta \hat{p}(\boldsymbol{r} ; \Gamma)\right\rangle_{\lambda_{t}}^{\mathrm{LG}},
\end{gathered}
$$

where $\delta \hat{p} \equiv \hat{\tau}^{a a(1)} / 3$. When $t$ is much larger than the correlation time of $\hat{\tau}^{a b(1)}$ and $\hat{q}^{a(1)}$, the transportation coefficients $\kappa, \eta$, and $\zeta$ depend on $t$ only through the $t$ dependence of $\beta_{t}$ and $\mu_{t}$, but are independent of $\boldsymbol{u}$. The hydrodynamic equation $\partial_{t} C_{t}^{\alpha}+\partial^{\alpha} J_{t}^{\alpha a(0)}=0$ is the NavierStokes equation, where $J_{t}^{4 a(1)}=0$. As is understood from the derivation method, the equation is valid up to $t \simeq O\left(\epsilon^{-2}\right)$, which is enough to describe relaxation processes to the global equilibrium state. The expression (12) with $\hat{\Sigma}_{t} \simeq O(\epsilon)$ implies that the distribution $P_{t}(\Gamma)$ is close to the local equilibrium in the relaxation processes.

It should be noted that the well-defined nature of the Green-Kubo formulas for nonuniform systems, (23), (24), and (25), remain to be studied seriously, in particular, with regard to their relevance to the power-law behavior of the time correlation functions in the formulas [27]. This is also related to the study of fluctuations of the macroscopic density fields defined as the average of $\hat{C}^{\alpha}$ over a spherical region with radius $\Lambda$, where $\Lambda$ satisfies $\xi_{\text {micro }} \ll \Lambda \ll \xi_{\text {macro }}$. Here, on one hand, because $\xi_{\text {micro }} \ll \Lambda$, it is expected from the law of large numbers that $\hat{C}_{\Lambda}^{\alpha}(r ; \Gamma)$ takes a typical value with respect to the probability density $P_{t}(\Gamma)$. On the other hand, the condition $\Lambda \ll \xi_{\text {macro }}$ leads to the result that the typical value of $\hat{C}_{\Lambda}^{\alpha}(\boldsymbol{r} ; \Gamma)$ is independent of the cutoff length $\Lambda$. In this sense, $\hat{C}_{\Lambda}^{\alpha}(\boldsymbol{r} ; \Gamma)$ defines macroscopic density fields without ambiguity. Since their typical value is expected to be given by $C_{t}^{\alpha}(\boldsymbol{r})$, the deterministic part of the evolution equation for the macroscopic density fields is the hydrodynamic equation. Constructing a simple formulation for describing statistical properties of $\hat{C}_{\Lambda}^{\alpha}(\boldsymbol{r} ; \Gamma)$ is the next natural problem.

Concluding remarks.-In summary, the Navier-Stokes equation has been derived from Hamiltonian particle systems in the most compact manner. The simple method presented in this Letter is expected to be useful for the derivation of hydrodynamic equations in other systems such as relativistic systems [28,29], anisotropic molecular systems [30], viscoelastic systems [31-33], dissipative particles [34], and active matter [35]. The formulation may also be developed so as to describe more complicated behavior near boundaries. It is stimulating to theoretically study a limit of the hydrodynamic nonslip boundary conditions [36].

Another important theoretical problem to be solved is to derive the hydrodynamic equations for other initial distributions. However, it seems quite difficult to study the 
problem, and a new concept would be necessary for a breakthrough. In particular, a role of the local equilibrium distributions may be clarified more. Regarding this difficulty, neither a proof of the nondecreasing property of $\mathcal{S}\left(C_{t}\right)$ nor a counterexample is obtained, whereas $\mathcal{S}^{(0)}\left(C_{t}\right)$ is found to be nondecreasing when the Navier-Stokes equation is considered. The understanding of $\mathcal{S}(C)$ beyond $\mathcal{S}^{(0)}(C)$ may be connected to steady-state thermodynamics [37-46].

I hope that the formulation described in this Letter will be developed further in order to discover the relationship between the macroscopic and microscopic descriptions.

The author thanks Y. Yokokura, M. Fukuma, and T. Kunihiro for stimulating discussions on the foundation of hydrodynamics. The author also thanks Y. Nakayama, T. Nakamura, Y. Oono, T. Ooshida, M. Otsuki, K. Saito, T. Sasamoto, N. Shiraishi, K. Takeuchi, H. Tasaki, and $\mathrm{H}$. Watanabe for useful comments on the manuscript. The present study was supported by KAKENHI No. 22340109 and No. 25103002, and by the JSPS Core-to-Core program "Non-equilibrium dynamics of soft-matter and information."

[1] L. D. Landau and E. M. Lifshitz, Fluid Mechanics, (Pergamon Press, Oxford, 1959).

[2] T. S. Komatsu, S. Matsumoto, T. Shimada, and N. Ito, [Int. J. Mod. Phys. C (to be published)].

[3] H. Grad, Phys. Fluids 6, 147 (1963).

[4] J. H. Irving and J. G. Kirkwood, J. Chem. Phys. 18, 817 (1950).

[5] C. B. Morray, Commun. Pure Appl. Math. 8, 279 (1955).

[6] H. Mori, Phys. Rev. 112, 1829 (1958).

[7] J. A. Mclennan, Phys. Fluids 3, 493 (1960); Introduction to Non-Equilibrium Statistical Mechanics (Prentice-Hall, Englewood Cliffs, NJ, 1988).

[8] D. N. Zubarev, Nonequilibrium Statistical Thermodynamics (Consultants Bureau, New York, 1974).

[9] K. Kawasaki and J. D. Gunton, Phys. Rev. A 8, 2048 (1973).

[10] C. Maes and K. Netočný, J. Math. Phys. (N.Y.) 51, 015219 (2010).

[11] H. Spohn, Large Scale Dynamics of Interacting Particles, Texts and Monographs in Physics (Springer-Verlag, Heidelberg, 1991).

[12] R. Esposito and R. Marra, J. Stat. Phys. 74, 981 (1994).

[13] A. Lenard, J. Stat. Phys. 19, 575 (1978).

[14] H. Tasaki, arXiv:cond-mat/0009206.
[15] C. Jarzynski, Phys. Rev. Lett. 78, 2690 (1997).

[16] D. J. Evans, E. G. D. Cohen, and G. P. Morriss, Phys. Rev. Lett. 71, 2401 (1993).

[17] G. Gallavotti and E. G. D. Cohen, Phys. Rev. Lett. 74, 2694 (1995).

[18] J. Kurchan, J. Phys. A 31, 3719 (1998).

[19] C. Maes, J. Stat. Phys. 95, 367 (1999).

[20] J. L. Lebowitz and H. Spohn, J. Stat. Phys. 95, 333 (1999).

[21] G. E. Crooks, Phys. Rev. E 61, 2361 (2000).

[22] C. Jarzynski, J. Stat. Phys. 98, 77 (2000).

[23] U. Seifert, Phys. Rev. Lett. 95, 040602 (2005).

[24] See Supplemental Material at http://link.aps.org/ supplemental/10.1103/PhysRevLett.112.100602 for explicit forms of the currents and the derivation of (18) and (19).

[25] S. Olla, S. R. S. Varadhan, and H. T. Yau, Commun. Math. Phys. 155, 523 (1993).

[26] M. S. Green, J. Chem. Phys. 22, 398 (1954).

[27] Y. Pomeau and P. Resibois, Phys. Rep. 19, 63 (1975).

[28] Y. Minami and Y. Hidaka, Phys. Rev. E 87, 023007 (2013).

[29] K. Tsumura and T. Kunihiro, Phys. Rev. E 87, 053008 (2013).

[30] P. C. Martin, O. Parodi, and P. S. Pershan, Phys. Rev. A 6, 2401 (1972).

[31] T. Ooshida and K. Sekimoto, Phys. Rev. Lett. 95, 108301 (2005).

[32] T. Azeyanagi, M. Fukuma, H. Kawai, and K. Yoshida, Phys. Lett. B 681, 290 (2009).

[33] M. Fukuma and Y. Sakatani, Phys. Rev. E 84, 026316 (2011).

[34] J. J. Brey, J. W. Dufty, C. S. Kim, and A. Santos, Phys. Rev. E 58, 4638 (1998).

[35] M. C. Marchetti, J. F. Joanny, S. Ramaswamy, T. B. Liverpool, J. Prost, M. Rao, and R. A. Simha, Rev. Mod. Phys. 85, 1143 (2013).

[36] Y. Zhu and S. Granick, Phys. Rev. Lett. 88, 106102 (2002).

[37] Y. Oono and M. Paniconi, Prog. Theor. Phys. Suppl. 130, 29 (1998).

[38] T. Hatano and S.-i. Sasa, Phys. Rev. Lett. 86, 3463 (2001).

[39] D. Ruelle, Proc. Natl. Acad. Sci. U.S.A. 100, 3054 (2003).

[40] S.-i. Sasa and H. Tasaki, J. Stat. Phys. 125, 125 (2006).

[41] T. S. Komatsu, N. Nakagawa, S.-i. Sasa, and H. Tasaki, Phys. Rev. Lett. 100, 230602 (2008).

[42] T. S. Komatsu, N. Nakagawa, S.-i. Sasa, and H. Tasaki, J. Stat. Phys. 142, 127 (2011).

[43] N. Nakagawa, Phys. Rev. E 85, 051115 (2012).

[44] C. Maes and K. Netočný, arXiv:1206.3423.

[45] L. Bertini, D. Gabrielli, G. Jona-Lasinio, and C. Landim, Phys. Rev. Lett., 110, 020601 (2013).

[46] S.-i. Sasa, J. Stat. Mech. (2014) P01004. 